\title{
PHENOMENOLOGY OF SPACE TIME FLUCTUATIONS
}

\author{
R. ALOISIO* \\ INFN - Laboratori Nazionali Gran Sasso \\ SS 17 bis, Assergi (AQ) Italy, \\ E-mail: roberto.aloisio@lngs.infn.it \\ P. BLASI \\ INAF - Osservatorio Astrofisico Arcetri \\ Largo E. Fermi 5, 50125 Firenze Italy \\ E-mail: blasi@arcetri.astro.it \\ A. GALANTE \\ Dipartimento di Fisica, Università di L'Aquila \\ Via Vetoio, 67100 Coppito (AQ) Italy \\ E-mail: angelo.galante@lngs.infn.it \\ A.F. GRILLO \\ INFN - Laboratori Nazionali Gran Sasso \\ SS 17 bis, Assergi (AQ) Italy, \\ E-mail: aurelio.grillo@lngs.infn.it
}

\begin{abstract}
Quantum gravitational effects may induce stochastic fluctuations in the structure of space-time, to produce a characteristic foamy structure. It has been known for some time now that these fluctuations may have observable consequences for the propagation of cosmic ray particles over cosmological distances. While invoked as a possible explanation for the detection of the puzzling cosmic rays with energies in excess of the threshold for photopion production (the so-called super-GZK particles), we demonstrate here that lower energy observations may provide strong constraints on the role of a fluctuating space-time structure. We note also that the same fluctuations, if they exist, imply that some decay reactions normally forbidden by elementary conservation laws, become kinematically allowed, inducing the decay of particles that are seen to be stable in our universe. Due to the strength of the prediction, we are led to consider this finding as the most severe constraint on the classes of models that may describe the effects of gravity on the structure of space-time. We also propose and discuss several potential loopholes of our approach, that may affect our conclusions. In particular, we try to identify the situations in which despite a fluctuating energy-momentum of the particles, the reactions mentioned above may not take place.
\end{abstract}

*invited speaker 


\section{Introduction}

In the last few years the hunt for possible minuscule violations of the fundamental Lorentz invariance (LI) has been object of renewed interest, in particular because it has been understood that cosmic ray physics has an unprecedented potential for investigation in this field 1234156 . Some authors 3417 have even invoked possible violations of LI as a plausible explanation to some puzzling observations related to the detection of ultra high energy cosmic rays (UHECRs) with energy above the so-called GZK feature $\frac{8}{\text {, }}$, and to the unexpected shape of the spectrum of photons with super-TeV energy from sources at cosmological distances.

Both types of observations have in fact many uncertainties, either coming from limited statistics of very rare events, or from accuracy issues in the energy determination of the detected particles, and most likely the solution to the alleged puzzles will come from more accurate observations rather than by a violation of fundamental symmetries.

For this reason, from the very beginning we proposed $[5]$ that cosmic ray observations should be used as an ideal tool to constrain the minuscule violations of LI, rather than as evidence for the need to violate LI. The reason why the cases of UHECRs and TeV gamma rays represent such good test sites for LI is that both are related to physical processes with a kinematical energy threshold, which is in turn very sensitive to the smallest violations of LI. UHECRs are expected to suffer severe energy losses due to photopion production off the photons of the cosmic microwave background (CMB), and this should suppress the flux of particles at the Earth at energies above $\sim 10^{20} \mathrm{eV}$, the so called GZK feature.

Present operating experiments are AGASA 9 and HiRes 10, and they do not provide strong evidence either in favor or against the detection of the GZK feature 11. A substantial increase in the statistics of events, as expected with the Auger project 12 and with EUSO 13 , should dramatically change the situation and allow to detect the presence or lack of the GZK feature in the spectrum of UHECRs. These are the observations that will provide the right ground for imposing a strong limit on violations of LI. For the case of $\mathrm{TeV}$ sources, the process involved is pair production 14 of high energy gamma rays on the photons of the infrared background. In both cases, a small violation of LI can move the threshold to energies which are smaller than the classical ones, or move them to infinity, making the reactions impossible. The detection of the GZK suppression or the cutoff in the gamma ray spectra of gamma ray sources at cosmological distances will prove that LI is preserved to correspondingly high accuracy $[5$.

The recipes for the violations of LI generally consist of requiring an explicit modification of the dispersion relation of high energy particles, due to their propagation in the "vacuum", now affected by quantum gravity (QG). This effect is generally parametrized by introducing a typical mass, expected to be of the order of the Planck mass $\left(M_{P}\right)$, that sets the scale for QG to become effective.

However, explicit modifications of the dispersion relation are not really neces- 
sary in order to produce detectable effects, as was recently pointed out in Refs. 15161718 for the case of propagation of UHECRs. It is in fact generally believed that coordinate measurements cannot be performed with precision better than the Planck distance (time) $\delta x \geq l_{P}$, namely the distance where the metric of space-time must feature quantum fluctuations. A similar line of thought implies that an uncertainty in the measurement of energy and momentum of particles can be expected, according with the relation $\delta p \simeq \delta E \simeq p^{2} / M_{P}$. As discussed also in Refs. $16 / 17$ the apparent problem of super-GZK particles may find a solution also in the context of this uncertainty approach.

We discuss here this appealing approach more in detail, by taking into account the effects of the propagation of CRs in the QG vacuum in the presence of the universal microwave background radiation. A fluctuating metric implies that different measurements of the particle energy or momentum may result in different outcomes. Therefore it becomes important to define the probability that the measured energy (momentum) of a particle is above some fixed value. Note that averaging over a large number of measurements would yield the classical values for the energy and momentum. The process of measurement mentioned above, during the propagation of particles over cosmological distances occurs at each single interaction of the particle with the environment. At each interaction vertex, the fluctuating energy/momentum of the particle is compared with the kinematic threshold for the occurrence of some physical process (in our case the photopion production). A clear consequence of this approach is that particles with classical energy below the standard Lorentz invariant threshold have a certain probability of interacting. In the same way, particles above the classical threshold have a finite probability of evading interaction. We show here that the most striking consequences of the approach described above derive from low energy particles rather than from particles otherwise above the threshold for photopion production.

However, the possibility of a fluctuating energy and momentum is mainly constrained by other processes that could arise. The fluctuations of energy and momentum are responsible, infact, for decaying processes otherwise impossible, typically prevented by energy and momentum conservation. These decaying processes represent the most stringent test of the proposed model. In the present paper we will discuss these decaying processes, showing how they could arise. From a general point of view a particle propagating in a fluctuating vacuum acquires an energy dependent fluctuating effective mass (the fluctuating dispersion relations introduced in 19. which may be responsible for kinematically forbidden decay reactions to become kinematically allowed.

If this happens, particles that are known to be stable would decay, provided no other fundamental conservation law is violated (e.g.: baryon number conservation, charge conservation). A representative example is that of the reaction $p \rightarrow p+$ $\pi^{0}$, that is prevented from taking place only due to energy conservation. With a fluctuating metric, we find that if the initial proton has energy above a few 
$10^{15} \mathrm{eV}$, the reaction above can take place with a cross section typical of hadronic interactions, so that the proton would rapidly lose its energy. Similar conclusions hold for the electromagnetic process $p \rightarrow p+\gamma$.

The fact that particles that would be otherwise stable could decay has been known for some time now 2021 and in fact it rules out a class of non-fluctuating modifications of the dispersion relations for some choices of the sign of the modification: the new point here is that it does not appear to be possible to fix the sign of the fluctuations, so that the conclusions illustrated above seem unavoidable. This result represents the most striking test of the fluctuating picture discussed in this paper and could in principle invalidate the basis of the proposed model itself.

The plan of the paper is the following: in $\S 2$ we discuss the effect of fluctuations on the propagation of high energy particles, setting also the computational framework of the paper. In $\S 3$, we discuss, mainly from the astrophysical point of view, the possibility of putting under experimental scrutiny some of the conclusions reached in $\S 2$. In section $\S 4$ we will discuss the decays of stable particles induced by fluctuations. Finally in section $\S 5$ we argue that the comparison of our predictions with experimental data indicates a strong inconsistency, implying that the framework of quantum fluctuations currently discussed in most literature is in fact ruled out. The strength of this conclusion leads us to try to identify possible loopholes in our working assumptions. The ways to avoid the dramatic effects of the fluctuating energy-momentum of a particle should be mainly searched in the dynamics of Quantum Gravity. These effects, in which our knowledge is poor to say the least, might forbid processes even when these processes are kinematically allowed due to the fluctuations in the energy and momentum.

\section{The effect of Space-Time fluctuations on the propagation of high energy particles.}

While electroweak and strong interactions propagate through space-time, gravity turns out to be a property of the space-time itself. This simple statement has profound implications in the quantization of gravity. Our belief that gravity can be turned into a quantum theory immediately implies that the structure of spacetime has quantum fluctuations itself. Another way of rephrasing this concept is that space-time is expected to have a granular (or foamy) structure, where however the size of space-time cells fluctuates stochastically, thereby causing an intrinsic uncertainty in the measurements of space-time lengths, and indirectly of energy and momentum of a particle moving through space-time. The uncertainty appears on scales comparable with the Planck scale (the quantization scale of gravity).

It is generally argued that measurements of distances (times) smaller than the Planck length (time) are conceptually unfeasible, since the process of measurement collects in a Planck size cell an energy in excess of the Planck mass, hence forming a black hole, in which information is lost. This can be translated in different ways into an uncertainty on energy-momentum measurements 1617. The Planck length 
is a good estimate of the uncertainty in the De Broglie wave-length $\lambda$ of a particle with momentum $p$. Therefore $\delta \lambda \approx l_{P}$, and $\delta p=\delta(1 / \lambda) \approx\left(p^{2} l_{P}\right)=\left(p^{2} / M_{P}\right)$.

Speculating on the exact characteristics of the fluctuations induced by QG is beyond the scope of the present paper, and it would probably be useless anyway, since the current status of QG approaches does not allow such a kind of knowledge. We decided then to adopt a purely phenomenological approach, in which some reasonable assumptions are made concerning the fluctuations in the fabric of space-time, and their consequences for the propagation of high energy particles are inferred. Comparison with experimental data then possibly constrains QG models.

Following [16, we assume that in each measurement:

- the values of energy (momentum) fluctuate around their average values (assumed to be the result theoretically recoverable for an infinite number of measurements of the same observable):

$$
\begin{aligned}
E & \approx \bar{E}+\alpha \frac{\bar{E}^{2}}{M_{P}} \\
p & \approx \bar{p}+\beta \frac{\bar{p}^{2}}{M_{P}}
\end{aligned}
$$

with $\alpha, \beta$ normally distributed variables and $p$ the modulus of the 3 momentum (for simplicity we assume rotationally invariant fluctuations);

- the dispersion relation fluctuates as follows:

$$
P_{\mu} g^{\mu \nu} P_{\nu}=E^{2}-p^{2}+\gamma \frac{p^{3}}{M_{P}}=m^{2}
$$

and $\gamma$ is again a normally distributed variable.

Ideally, QG should predict the type of fluctuations introduced above, but, as already stressed, this is currently out of reach, therefore we assume here that the fluctuations are gaussian. Our conclusions are however not sensitive to this assumption: essentially any symmetrical distribution with variance $\approx 1$, within a large factor, would give essentially the same results. Furthermore we assume that $\alpha, \beta$ and $\gamma$ are uncorrelated random variables; again, this assumption reflects our ignorance in the dynamics of QG

The fluctuations described above will in general derive from metric fluctuations of magnitude $\delta g^{\mu \nu} \sim h^{\mu \nu} \frac{l_{P}}{l}$ 3117. Our assumption reflects the fact that, while the magnitude of the fluctuation can be guessed, we do not make any assumption on its tensorial structure $h^{\mu \nu}$.

Our interest will be now concentrated upon processes of the type

$$
a+b \rightarrow c+d
$$

where we assume that a kinematic threshold is present; in the realm of UHECR physics $(\mathrm{a}, \mathrm{b})$ is either $\left(\gamma, \gamma_{3 K}\right)$ or $\left(p, \gamma_{3 K}\right)$ and $(\mathrm{c}, \mathrm{d})$ is $\left(e^{+}, e^{-}\right)$or $(N, \pi)$. 
To find the value of initial momenta for which the reaction occurs we write down energy-momentum conservation equations and solve them with the help of the dispersion relations, as discussed in detail in 5 .

The energy momentum conservation relations are (in the laboratory frame, and specializing to the case in which the target (b) is a low energy background photon for which fluctuations can be entirely neglected)

$$
\begin{gathered}
E_{a}+\alpha_{a} \frac{E_{a}^{2}}{M_{P}}+\omega=E_{c}+\alpha_{c} \frac{E_{c}^{2}}{M_{P}}+E_{d}+\alpha_{d} \frac{E_{d}^{2}}{M_{P}} \\
p_{a}+\beta_{a} \frac{p_{a}^{2}}{M_{P}}-\omega=p_{c}+\beta_{c} \frac{p_{c}^{2}}{M_{P}}+p_{d}+\beta_{d} \frac{p_{d}^{2}}{M_{P}} .
\end{gathered}
$$

These equations refer to head-on collisions and collinear reaction products, which is appropriate for threshold computations. Together with the modified dispersion relations, these equations, after some manipulations, lead to a cubic equation for the initial momentum as a function of the momentum of one of products, and, after minimization, they define the threshold for the process considered. In figure 1 we report the distribution of thresholds in the $\approx 70 \%$ of cases in which the solution is physical; in the other cases the kinematics does not allow the reaction.

This threshold distribution can be interpreted in the following way: a particle with energy above $\sim 10^{15} \mathrm{eV}$ has essentially $70 \%$ probability of being above threshold, and therefore to be absorbed. In the other $30 \%$ of the cases the protons do not interact.

In (45) the fluctuations are taken independently for each particle, which is justified as long as the energies are appreciably smaller than the Planck energy. At that point it becomes plausible that different particles experience the same fluctuations, or more precisely fluctuations of the same region of space-time. It is instructive to consider this case in some more detail: we introduce then the four-momenta (and dispersion relations) of all particles fluctuating in the same way. Specializing to proton interaction on CMBR, the equation which defines the threshold $p_{t h}$ is $\underline{5}$.

$$
\eta \frac{2 p_{0}^{3}}{\left(m_{\pi}^{2}+2 m_{\pi} m_{p}\right) M_{P}} \frac{m_{\pi} m_{p}}{\left(m_{\pi}+m_{p}\right)^{2}}\left(\frac{p_{t h}}{p_{0}}\right)^{3}+\left(\frac{p_{t h}}{p_{0}}\right)-1=0
$$

where $\eta$ is a gaussian variable with zero average and variance of the order of (but not exactly equal to) one, and $p_{0}$ is the L.I. threshold (GZK). The threshold is the positive solution of this equation. 


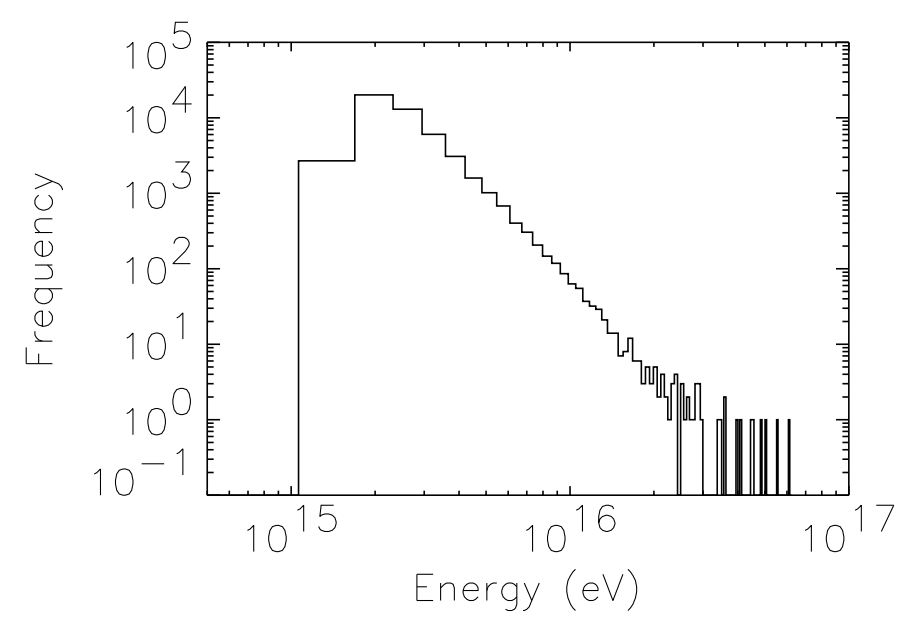

Figure 1. Threshold distribution for $p \gamma_{3^{\circ} K} \rightarrow N \pi$. In the $30 \%$ of cases the reaction is not allowed.

The coefficient of the cubic term is very large, of the order of $10^{13}$ in this case, so that unless $\eta$ is $O\left(10^{-13}\right)$, we can write, neglecting pion mass

$$
p_{t h} \approx p_{0}\left(\frac{m_{p}^{2} M_{P}}{\eta p_{0}^{3}}\right)^{\frac{1}{3}} .
$$

When $\eta$ becomes negative, the above equation has no positive root; this happens essentially in $50 \%$ of the cases. Since the gaussian distribution is flat in a small interval around zero, the distribution of thresholds for positive $\eta$ peaks around the value for $\eta \approx 1$, meaning that the threshold moves almost always down to a value of $\approx 10^{15} \mathrm{eV}$; , essentially the same result holds for fluctuations affecting only the incident (highest energy) particle. For independent fluctuations of final momenta, the asymmetry in the probability distribution of allowed thresholds arises from the fact that even exceedingly small negative values of the fluctuations lead to unphysical solutions.

Building upon our findings, we now apply the same calculations to the case of UHECR protons propagating on cosmological distances. An additional ingredient is needed to complete the dynamics of the process of photopion production, namely the cross section. The rather strong assumption adopted here is that the cross section remains the same as the Lorentz invariant one, provided the reaction is kinematically allowed. This implies that the interaction lengths remain unchanged.

In order to assess the situation of UHECRs, we first consider the case of particles above the threshold for photopion production in a Lorentz invariant world. According with eqs. (4 5), in this case particles have a probability of $\approx 30 \%$ of being not kinematically allowed to interact inelastically with a photon in the CMBR. 
Therefore, if our assumption on the invariance of the interaction length is correct, then each proton is still expected to make photopion production, although with a slightly larger pathlength.

The situation is however even more interesting for particles that are below the Lorentz invariant threshold for the process of photopion production. If the energy is below a few $10^{18} \mathrm{eV}$, a galactic origin seems to be in good agreement with measurements of the anisotropy of cosmic ray arrival directions 2223 . We will not consider these energies any longer. On the other hand, at energies in excess of $10^{19}$ $\mathrm{eV}$, cosmic rays are believed to be extragalactic protons, mainly on the ground of the comparison of the size of the magnetized region of our Galaxy and the Larmor radius of these particles. We take these pieces of information as the basis for our line of thought. If the cosmic rays observed in the energy range $E>10^{19} \mathrm{eV}$ are extragalactic protons, then our previous calculations apply and we may expect that these particles have $\mathrm{a} \sim 70 \%$ probability of suffering photopion production at each interaction with the CMB photons, even if their energy is below the classical threshold for this process. Note that the pathlength associated with the process is of the order of the typical pathlength for photopion production (a few tens of $\mathrm{Mpc}$ ), therefore we are here discussing a dramatic process in which the absorption length of particles drops from Gpc, which would be pertinent to particles with energy below $\sim 10^{20} \mathrm{eV}$ in a Lorentz invariant world, to several Mpc, with a corresponding suppression of the flux. What are the consequences for the observed fluxes of cosmic rays? The above result implies that all protons with $E>10^{15} \mathrm{eV}$ are produced within a radius of several tens of $\mathrm{Mpc}$, and above this energy there is no dramatic change of pathlength with energy. There is no longer anything special about $E \sim 10^{20} \mathrm{eV}$, and any mechanism invoked to explain the flux of super-GZK particles must be at work also at lower energies.

The basic situation remains the same in the case of pair production as the physical process under consideration. For a source at cosmological distance, a cutoff is expected due to pair production off the far infrared background (FIR) or the microwave background. Using the results in $[5]$ we expect that the modified thresholds are a factor $0.06(0.73)$ lower than the Lorentz invariant ones for the case of interaction on the CMBR (FIR). There is also a small increase in the pathlengths above the threshold, which would appear exponentially in the expression for the flux. Therefore there are two effects that go in opposite directions: the first moves the threshold to even lower energies, and the second increases the flux of radiation at Earth because of the increase of the pathlength. It seems that geometry fluctuations do not provide an immediate explanation of the possible detection of particles in excess of the expected ones from distance sources in the $\mathrm{TeV}$ region. In any case the experimental evidence for such an excess seems at present all but established. 


\section{Astrophysical observations}

As discussed in the previous section fluctuations in the space-time metric may induce a violation of Lorentz invariance that changes the thresholds for the photopion production of a very high energy proton off the photons of the CMBR, or for the pair production of a high energy gamma ray in the bath of the FIR or CMBR photons.

For the case of UHECRs interacting with the CMBR, we obtained a picture that changes radically our view of the effect of QG on this phenomenon, as introduced in previous papers: not only particles with energy above $\sim 10^{20} \mathrm{eV}$ are affected by the fluctuations in space-time, but also particles with lower energy, down to $\sim 10^{15} \mathrm{eV}$ seem to be affected by such fluctuations. In fact the latter, as a result of a fluctuating space-time, may end up being above the threshold for photopion production, so that particles may suffer significant absorption. Our conclusion is that all particles with energy in excess of $\sim 10^{15} \mathrm{eV}$ eventually detected at Earth would be generated at distances comparable with the pathlength for photopion production $(\sim 100 \mathrm{Mpc})$. A consequence of this is that there is no longer anything special characterizing the energy $\sim 10^{20} \mathrm{eV}$.

Since the conclusion reached in the previous section is quite strong, it is important to summarize in detail some tests that may allow to understand whether the current or future astrophysical observations are compatible with the scenario discussed in this paper.

a) Future experiments 1213 dedicated to the detection of UHECRs will provide a substantial increase in the statistics, so that the spectral features of the UHECRs in the energy region $E>10^{19} \mathrm{eV}$ can be resolved, and further indications on the nature of primaries and their possible extragalactic origin will be obtained. In particular the present possible disagreement between AGASA 24 and HiRes ${ }^{25]}$ will be clarified.

One should also keep in mind that an evaluation of the expected flux in terms of sources distributed as normal galaxies is in contradiction with AGASA data by an amount ranging from 2 to $6 \sigma$ depending on the assumed source spectrum 26]. Since the nature of the sources is not known, it is not clear if their abundance within the absorption pathlength is sufficient to explain the observed flux in presence of space-time fluctuations, nor if they can induce observable anisotropies.

In any case, in a Lorentz invariant framework a suppression in the flux at $\sim 10^{20}$ $\mathrm{eV}$ is expected. If such a feature is unambiguously detected in the UHECR spectrum, no much room would be left for the fluctuations of space-time discussed in this paper, since in this scenario nothing special happens around $10^{20} \mathrm{eV}$. In quantitative terms $[5]$ this would imply a phenomenological bound on $l_{P}$ now interpreted as a parameter: $l_{P}<10^{-46} \mathrm{~cm}$ instead of $l_{P} \approx 10^{-33} \mathrm{~cm}$; in other words, only fluctuations with variance $\approx 10^{-13}$, instead of 1 , would be allowed ${ }^{\text {a }}$

${ }^{a}$ Alternatively, one can assume a more general form of fluctuations, i.e. $\delta E \approx E\left(E / M_{P}\right)^{\alpha}$ and 
b) According with our findings, all particles with energy in excess of $\sim 10^{15} \mathrm{eV}$ lose their energy by photopion production on cosmological spatial scales, as a result of the metric fluctuations. This energy ends up mainly in gamma rays, neutrinos and protons. The protons pile up in the energy region right below $\sim 10^{15} \mathrm{eV}$. The gamma ray component actually generates an electromagnetic cascade that ends up contributing low energy gamma rays, in the energy band accessible to instruments like EGRET 28 and GLAST 29. This cascade flux cannot be larger than the measured electromagnetic energy density in the same band $\omega_{c a s}^{e x p}=10^{-6} \mathrm{eV} / \mathrm{cm}^{3}$ 28. The cascade flux in our scenario can be estimated as follows. Let $\Phi(E)=$ $\Phi_{0}\left(E / E_{0}\right)^{-\gamma}$ be the emissivity in UHECRs (particles $/ \mathrm{cm}^{3} / \mathrm{s} / \mathrm{GeV}$ ). Let us choose the energy $E_{0}=10^{10} \mathrm{GeV}$ and let us normalize the flux to the observations at the energy $E_{0}$. The total energy going into the cascade can be shown to be

$$
\omega_{\text {cas }} \approx \frac{5 \times 10^{-4}}{\gamma-2} x_{\min }^{2-\gamma} \xi \mathrm{eV} \mathrm{cm}{ }^{-3}
$$

where $\xi$ is the fraction of energy going into gamma rays in each photopion production, and $x_{\text {min }}=\left(E_{t h} / E_{0}\right)=10^{-4}$ for $E_{t h}=10^{15} \mathrm{eV}$. It is easy to see that, for $\gamma=2.7$, the cascade bound is violated unless $\xi \ll 10^{-3}$.

One note of warning has to be sent concerning the development of the electromagnetic cascade: the same violations of LI discussed here affect other processes, as stressed in the paper. For instance pair production and pion decay are also affected by violations of LI 30 . Therefore the possibility that the cascade limit is exceeded concerns only those scenarios of violations of LI that do not inhibit appreciably pair production and the decay of neutral pions.

The protons piled up at energies right below $10^{15} \mathrm{eV}$, would be a nice signature of this scenario, but it seems difficult to envision a way of detecting these remnants. In fact, even a tiny magnetic field on cosmological scales would make the arrival time of these particles to Earth larger than the age of the universe. Moreover, even assuming an exactly zero extragalactic magnetic field, these particles need to penetrate the magnetic field of our own Galaxy and mix with the galactic cosmic rays, making their detection extremely problematic if not impossible.

Clearly a more detailed flux computation, taking into account propagation of primaries as well as generation and propagation of the secondaries is needed in order to assess in a more quantitative way observable effects of possible metric fluctuations on UHECRs.

Let us conclude this section sending a note of warning concerning Eq. (17), in this expression the dependence on the CMB photon energy is washed out by the approximation done (we have neglected the pion mass). From the physical point of view this corresponds to the appearence of an effective mass (momentum dependent) of the proton due to the effect of fluctuations. The effective mass of

similar for momentum and dispersion relations 27. In this case the basic conclusions reached here remain unchanged. 
the proton may be responsible for the decay of this particle. As we will discuss in the next section the possibility of a decaying proton is a very stringent test for the fluctuations picture much powerful than the astrophysical observations discussed in the present section.

\section{Decay of stable particles}

Let us discuss in this section the most striking test of the models that predict energy and momentum fluctuations. We will discuss here the possibility that these fluctuations may induce particles decays otherwise impossible. This possibility, already discussed in the framework of non-fluctuating modifications of the dispersion relation 2021, could in principle rule out the models with fluctuations. In this section we will discuss the basic features of the decays, leaving a detailed discussion of the implications and possible way out to the next section.

We will consider three specific decay channels, that illustrate well, in our opinion, the consequences of the quantum fluctuations introduced above. We start with the reaction

$$
p \rightarrow p+\pi^{0}
$$

and we denote with $p\left(p^{\prime}\right)$ the momentum of the initial (final) proton, and with $k$ the momentum of the pion. Clearly this reaction cannot take place in the reality as we know it, due to energy conservation. However, since fluctuations have the effect of emulating an effective mass of the particles, it may happen that for some realizations, the effective mass induced to the final proton is smaller than the mass of the proton in the initial state, therefore allowing the decay from the kinematical point of view. Since no conservation law or discrete symmetry is violated in this reaction, it may potentially take place. For the sake of clarity, it may be useful to invoke as an example the decay of the $\Delta^{+}$resonance, which is structurally identical to a proton, but may decay to a proton and a pion according to the reaction $\Delta^{+} \rightarrow$ $p+\pi^{0}$, since its mass is larger than that of a proton. From the physical point of view, the effect of the quantum fluctuations may be imagined as that of exciting the proton, inducing a mass slightly larger than its own (average) physical mass.

Following the discussion of the previous sections we expect to find that for momenta above a given threshold, depending on the value of the random variables, the decay may become kinematically allowed. In general, the probability for this to happen has to be calculated numerically from the conservation equations supplemented by the dispersion relations 31 .

Although a full calculation is possible, it is probably more instructive to proceed in a simplified way, in which only the fluctuations in the dispersion relation of the particle in the initial state are taken into account. Neglecting the corresponding fluctuations in the final state should not affect the conclusions in any appreciable way, unless the fluctuations in the initial and final states are correlated (we will return to this possibility at the end of section $\S 5$ ). 
In this approximation, the threshold for the process of proton decay to a proton and a neutral pion can be written as follows (neglecting corrections to order higher than $\left.p / M_{P}\right)$ :

$$
\gamma \frac{2 p_{t h}^{3}}{M_{P}}-2 m_{\pi} m_{p}-m_{\pi}^{2}=0
$$

with solution

$$
p_{t h}=\left(\frac{\left(2 m_{p} m_{\pi}+m_{\pi}^{2}\right) M_{P}}{2 \gamma}\right)^{\frac{1}{3}} .
$$

For negative values of $\gamma$, the above equation has no positive root; this happens in $50 \%$ of the cases. Since the gaussian distribution is essentially flat in a small interval around zero, the distribution of thresholds for positive $\gamma$ (i.e. in the remaining 50 $\%$ of the cases) peaks around the value for $\gamma \approx 1$, meaning that the threshold moves almost always down to a value of $\approx 10^{15} \mathrm{eV} \underline{1519}$; essentially the same result holds for generic fluctuations (i.e. not confined to the dispersion relations) affecting only the incident particle, namely the one with the highest energy 31 .

The reason why the effects of fluctuations are expected to occur at such low energies is that starting from that energy region the fluctuation term becomes comparable with the rest mass of the particle. In fact the same concept of rest mass of a particle may lose its traditional meaning at sufficiently high energies 18 .

It can be numerically confirmed that independent fluctuations of momenta (and/or of the dispersion relations) of the decay products are more likely to make the decay easier rather than more difficult, due to the non linear dependence of the threshold on the strength of fluctuations: the probability that the decay does not take place is in fact $\approx 30 \%$. In the remaining cases, the decay will occur if the momentum of the initial proton is larger than $p_{t h}$. The distribution of $p_{t h}$ is essentially identical to the one reported in $\S 2$ for the photopion production.

All the discussion reported so far remains basically unchanged if similar reactions are considered. For instance the reaction $p \rightarrow \pi^{+} n$ is kinematically identical to the one discussed above. For all these reactions, we expect that once they become kinematically allowed, the energy loss of the parent baryon is fast. For the case of nuclei, all the decays that do not change the nature of the nucleon leave $(\mathrm{A}, \mathrm{Z})$ unchanged, so we do not expect any substantial blocking effect in nuclei.

Another reaction that may be instructive to investigate is the spontaneous pair production from a single photon, namely 31

$$
\gamma \rightarrow e^{+} e^{-}
$$

In this case, following the calculations described above, we obtain the following expression for the threshold:

$$
p_{t h}^{\prime}=\left(\frac{4 m_{e}^{2} M_{P}}{2 \gamma^{\prime}},\right)^{\frac{1}{3}}
$$


and $p_{t h}^{\prime}$ is of the order of $10^{13} \mathrm{eV}$. Again, if the reaction becomes kinematically allowed, there does not seem to be any reason why the reaction should not take place with a rate dictated by the typical cross section of electromagnetic interactions.

Finally, we propose a third reaction that in its simplicity may represent the clearest example of reactions that should occur in a world in which quantum fluctuations behave in the way described above. Let us consider a proton that moves in the vacuum with constant velocity, and let us consider the elementary reaction of spontaneous photon emission. In the Lorentz invariant world the process of photon emission is known to happen only in the presence of an external field that may provide the conditions for energy and momentum conservation. However, in the presence of quantum fluctuations, one can think of the gravitational fluctuating field as such an external field, so that the particle can in fact radiate a photon without being in the presence of a nucleus or some other external recognizable field. The threshold for this process, calculated following the above procedure, is

$$
p_{t h}^{\prime \prime} \approx\left(\frac{m^{2} M_{P} \omega}{\gamma^{\prime \prime}}\right)^{\frac{1}{4}}
$$

where $\omega$ is the energy of the photon. This threshold approaches zero when $\omega \rightarrow 0$ : for instance, if $\omega=1 \mathrm{eV}$, then $p_{t h} \approx 300 \mathrm{GeV}$ for protons and $p_{t h} \approx 45 \mathrm{GeV}$ for electrons. In other words there should be a sizable energy loss of a particle in terms of soft photons. This process can be viewed as a sort of bremsstrahlung emission of a charged particle in the presence of the (fluctuating) vacuum gravitational potential.

Based on the arguments provided in this section, it appears that all particles that we do know are stable in our world, should instead be unstable at sufficiently high energy, due to the quantum fluctuations described above. In the next section we will take a closer look at the implications of the existence of these quantum fluctuations, and possibly propose some plausible avenues to avoid these dramatic conclusions.

\section{Discussion and Outlook}

If the decays discussed in the previous section could take place, our universe, at energies above a few $\mathrm{PeV}$ or even at much lower energies might be unstable, nothing like what we actually see. The decays

$$
\text { nucleon } \rightarrow \text { nucleon }+\pi
$$

would start to be kinematically allowed at energies that are of typical concern for cosmic ray physics, while the spontaneous emission of photons in vacuum might even start playing a role at much lower energies, testable in laboratory experiments. Without detailed calculations of energy loss rates it is difficult to assess the experimental consequences of this process.

For the nucleon decay, the situation is slightly simpler if we assume that the quantum fluctuations affect only the kinematics but not the dynamics, an assumption also used in in the photopion production study [19. In this case one would 
expect the proton to suffer the decay to a proton and a pion on a time scale of the same order of magnitude of typical decays mediated by strong interactions. This would basically cause no cosmic ray with energy above $\sim 10^{15} \mathrm{eV}$ to be around, something that appears to be in evident contradiction with observations ${ }^{\mathrm{b}}$.

In the following we will try to provide a plausible answer to these three very delicate questions:

(1) If the particles were kinematically allowed to decay, and there were no fundamental symmetries able to prevent the decay, would it take place?

(2) Is the form adopted for the quantum fluctuations correct and if so, how general is it?

(3) If in fact the form adopted for the fluctuations is correct, how general and unavoidable is the consequence that (experimentally) unobserved decays should take place?

Although the result that particles are kinematically allowed to decay is fairly general, the (approximate) lack of relativistic invariance forbids the computation of life-times ${ }^{c}$. Two comments are in order: first, the phase space for the decays described above, as calculated in the laboratory frame, is non zero and in fact it increases with the momentum of the parent particle. The effect of fluctuations can be seen as the generation of an effective (mass) $)^{2} \propto p^{3} / M_{P}$. A similar effect, although in a slightly different context, was noted in 4 .

Second, we do not expect dynamics to forbid the reactions: one must keep in mind that we are considering very small effects, at momenta much smaller than the Planck scale. For instance the gravitational potential of the vacuum fluctuations is expected to move quarks in a proton to excited levels, not to change its content, nor the properties of strong interactions.

There is a subtler possibility, which must be taken very seriously in our opinion, since it might invalidate completely the line of thought illustrated above, namely that the quantum fluctuations of the momenta of the particles involved in a reaction occur on time scales that are enormously smaller than the typical interaction/decay times. This situation might resemble the so called Quantum Zeno paradox, where continuously checking for the decay of an unstable particle effectively impedes its decay. This possibility is certainly worth a detailed study, that would however force one to handle the intricacies of matter in a Quantum Gravity regime. We regard this possibility as the most serious threat to the validity of the arguments in favor of quantum fluctuations discussed in this paper and in many others before it.

Let us turn out attention toward the question about the correctness and gener-

\footnotetext{
${ }^{\mathrm{b}}$ From a phenomenological point of view, consistency with experiments would require either that the variance of the fluctuations considered above is ridiculously small $\left(<10^{-24}\right)$ or, allowing more generic fluctuations $\Delta l \propto l_{P}\left(l_{P} / l\right)^{\alpha}$, that a fairly large value for $\alpha$ should be adopted 19 .

${ }^{\mathrm{c}}$ In fact life-times can be in principle estimated in approaches in which it is possible to make transformations between frames 183233 , despite the lack of LI.
} 
ality of the form adopted for the momentum fluctuations. It is generally accepted that the geometry of space-time suffers profound modifications at length (time) scales of the order of the Planck length (time), and that this leads to the emergence of a minimum measurable length. This may be reflected in a non commutativity of space-time and in a generalized form of the uncertainty principle.

The transition from uncertainty in the length or time scales to uncertainty in momenta of particles is undoubtedly more contrived and deserves some attention. The expressions in Eqs. (12) and (3) have been motivated in various ways 1516191834 in previous papers. For instance, the condition $\Delta l \geq l_{P}$ seems to imply the following constraint on wavelengths $\Delta \lambda \geq l_{P}$, otherwise it would be possible to design an experimental set-up capable of measuring distances with precision higher than $l_{P}$. Therefore $\Delta p \propto \Delta\left(\lambda^{-1}\right) \propto l_{P} p^{2}$. Similar arguments have been proposed, all based to some extent on the de Broglie relation $p \propto \lambda^{-1}$.

There is certainly no guarantee that the de Broglie relation continues to keep its meaning in the extreme conditions we are discussing, in particular in models in which the coordinates and coordinate-momentum commutators are modified with respect to standard quantum mechanics and the representation of momentum in terms of coordinate derivatives generally fails. For instance in a specific (although non-relativistic) example 35 the existence of a minimum length is shown to imply that

$$
p=\frac{2}{\pi l_{P}} \tan \left(\frac{\pi l_{P}}{2 \lambda}\right) .
$$

In other words, the de Broglie relation may be modified in such a way that a minimum wavelength corresponds to an unbound momentum. Notice, however, that we are considering here the effects of these modifications at length scales much larger than the Planck scale, where the correction is likely to be negligible. In general, if $p \propto \lambda^{-1} g\left(l_{P} / \lambda\right)$ then $\Delta p \propto l_{P} p^{2}+p O\left(l_{P}^{2} p^{2}\right)$. Hence, we do not expect that the result shown in the previous Section is appreciably modified.

Last but not least we notice that the fluctuations in the dispersion relations can be easily derived from fluctuations of the (vacuum) metric in the form given in 34 .

$$
d s^{2}=(1+\phi) d t^{2}-(1+\psi) d \mathbf{r}^{2}
$$

where $\phi, \psi$ are functions of the position in space-time.

The fluctuations of the dispersion relation, Eq. (3), follow if $\phi \neq \psi$ (i.e. non conformal fluctuations), assuming at least approximate validity of the de Broglie relation; if $\phi=\psi$ a much milder modification $\left(\mathrm{O}\left(\mathrm{pm}^{2} / M_{P}\right)\right)$ follows.

Having given plausibility arguments in favor of the form adopted for the fluctuations, at least for the case of non conformal fluctuations, we are left with the goal of proving an answer to the last question listed above, namely does a decay actually occur once it is kinematically allowed? Certainly the answer is positive if one continues to assume momentum and energy conservation, and modifications of these conservation laws with random terms of order $O\left(p^{2} / M_{P}\right)$ do not change 
this conclusion. The question then is whether we are justified in assuming energy and momentum conservation in the form used above. For instance, in the so-called Doubly Special Relativity (DSR) 32, theories and in general in models with deformed Poincare' invariance, the conservation relations may be modified in a non trivial, non additive and non abelian way. For instance, in the case of proton decay considered above, momentum conservation may read as 32.33

$$
\mathbf{p}_{p} \approx \mathbf{p}_{p}^{\prime}+\left(1+l_{P} E_{p}^{\prime}\right) \mathbf{p}_{\pi} \quad \text { or } \quad \mathbf{p}_{p} \approx \mathbf{p}_{\pi}^{\prime}+\left(1+l_{P} E_{\pi}^{\prime}\right) \mathbf{p}_{p}
$$

This certainly makes the probability of being above threshold smaller. However in order to qualitatively modify our results this probability should be in fact vanishingly small. For the case of low energy cosmic rays, this probability should be of the order of a typical decay time divided by the residence time of cosmic rays (mostly galactic at these energies) in our Galaxy.

We are led to conclude that allowing for modifications of the conservation relations does not appear to improve the situation to the point that the strong conclusions derived in the previous section can be avoided. In the same perspective, cancellation between fixed modifications of the dispersion relation and fluctuations (of the same order of magnitude) does not seem a viable way to proceed.

It is important however to notice that we have considered the above fluctuations as independent. In a full theory one should take into account possible correlations between fluctuations. The effect of correlations is very important because it pushes to higher energies the fluctuation scale of the particle momentum (energy). Let us discuss in more detail this point. Quantum fluctuations of the momenta of the particles involved in a reaction occour on time scales that are much smaller than the typical interaction time. Particles during the interaction time experience a large number of fluctuations, typically

$$
N=\frac{\tau}{\tau_{P}}=\frac{1}{p \tau_{P}}=\frac{M_{P}}{p},
$$

where we have used $\tau \sim 1 / p$ for the interaction time scale and $\tau_{P} \sim 1 / M_{P}$ for the fluctuation time scale. Assuming independent fluctuations of energy and momentum the fluctuation variance $\sigma$ will be

$$
\sigma^{2}=\frac{p^{3}}{M_{P} \sqrt{N}}=\frac{p^{3}}{M_{P}}\left(\frac{p}{M_{P}}\right)^{1 / 2}
$$

and the fluctuation variance becomes of the order of the proton mass $\sigma \simeq m_{p}$ already at momentum $p \simeq 10^{17} \mathrm{eV}$. In this case the situation resembles as discussed above and, for instance, the decaying of the proton arises already at lower energies. Let us consider now the case in which there is some degree of correlation in the momentum (energy) fluctuations. In this case the fluctuation variance $\sigma$ will be 


$$
\sigma^{2}=\frac{p^{3}}{M_{P} N^{\alpha}}=\frac{p^{3}}{M_{P}}\left(\frac{p}{M_{P}}\right)^{\alpha},
$$

where we have introduced the exponent $\alpha>1 / 2$ that parametrizes the effect of correlations. In this case the fluctuation variance becomes of the order of the proton mass at larger energies, namely $\sigma \simeq m_{p}$ at momentum of the order of

$$
p \simeq M_{P}\left(\frac{m_{p}}{M_{P}}\right)^{\frac{2}{3+\alpha}} .
$$

A detailed analysis of possible correlations between fluctuations, namely an analytic determination of $\alpha$, is impossible at this stage because it implies a better knowledge of the theory, and in particular of the dynamics of the QG regime.

Finally, a separate discussion is needed for those theories that include the relativity principle (exemplified by DSR models). The DSR theories are characterized by an extended Lorentz invariance 32 with two separate invariant scales: the light velocity and the Planck length. Moreover, in the low energy limit of DSR, or for distances much larger than the Planck length, the usual Lorentz invariance is recovered.

Using these two characteristics of the DSR theories it is easy to proove that particle kinematics in DSR is the same as in the usual Lorentz invariant theories. This result holds in the case in which there are no fluctuations of energy and momentum. In the most general case in which fluctuations of energy and momentum are taken into account it is difficult to prove that the situation remains unchanged. Nevertheless, if in DSR the relativity principle remains at work also in the fluctuating case the DSR approach seems the most promising in order to escape the particles decays discussed in this paper that seems to invalidate all the other models.

\section{References}

1. D.A. Kirzhnits and V.A. Chechin, Sov. Jour. Nucl. Phys. 15, 585 (1971).

2. L. Gonzalez-Mestres, Proc. 26th ICRC (Salt Lake City, USA), 1, 179 (1999).

3. G. Amelino Camelia, J. Ellis, N.E. Mavromatos and S. Sarkar Nature 393 (1998) 763.

4. S. Coleman and S.L. Glashow, Phys. Rev. D59, 116008 (1999).

5. R. Aloisio, P. Blasi, P.L. Ghia and A.F. Grillo, Phys. Rev. D62, 053010 (2000).

6. J.M. Carmona, J.L. Cortes, J. Gamboa and F. Mendez, preprint hep-th/0301248

7. O. Bertolami and C.S. Carvalho, Phys. Rev. D61, 103002 (2000); O. Bertolami, preprint astro-ph/0012462

8. K. Greisen, Phys. Rev. Lett. 16, 748 (1966); G.T. Zatsepin and V.A. Kuzmin, Pis'ma Zh. Ekps. Teor. Fiz. 4, 114 (1966) [JETP Lett. 4, 78 (1966)].

9. N. Hayashida et al. [AGASA collaboration], Phys. Rev. Lett. 73, 3491 (1994).

10. T. Abu-Zayyad et al. [Hires collaboration], astro-ph/0208243

11. D. De Marco, P. Blasi and A.V. Olinto, Astrop. Phys. 20, 53 (2003).

12. J. Blümer et al. [Auger Collaboration], J. Phys. G29 867 (2003).

13. EUSO space Observatory, http://www.euso-mission.org 
14. A.I. Nikishov, Sov. Phys. - JETP 14, 393 (1962); P. Goldreich and P. Morrison, Sov. Phys. - JETP 18, 239 (1964); R.J. Gould and G.P. Schreder, Phys. Rev. Lett. 16, 252 (1966).

15. L.H. Ford Int. J. Theor. Phys. 382941 (1999).

16. Y.J. Ng, D.S. Lee, M.C. Oh and H. van Dam, Phys. Lett. B507 236 (2001).

17. Y.J. Ng, Int. J. Mod. Phys. D11 1585 (2002) (preprint gr-qc/0201022).

18. R. Lieu, Astrophys. J. 568 L67 (2002).

19. R. Aloisio, P. Blasi, A. Galante, P.L. Ghia and A.F. Grillo, Astrop. Phys. 19, 127 (2003).

20. L. Gonzalez-Mestres, preprint hep-ph/9905430

21. T. Jacobson, S. Liberati and D. Mattingly, Phys. Rev. D66, 081302 (2002).

22. N.Hagashida et al., Astrop. Phys. 10303 (1999).

23. D.J. Bird et al., Ap. J. 511739 (1999).

24. N. Sakay et al. Proceedings of 2001 ICRC.

25. C.C.H. Jui et al., Proceedings of 2001 ICRC.

26. M. Blanton, P. Blasi and A.V. Olinto, Astrop. Phys. 15, 275 (2001).

27. M. Jankiewicz, T.W. Kephart and T.J. Weiler, preprint hep-ph/0312221

28. P. Sreekumar et al. [EGRET collaboration], Astroph. J. 494 (1998) 523.

29. GLAST Tlescope, http://www-glast.stanford.edu.

30. G. Amelino-Camelia, Phys. Lett. B528 (2002) 181.

31. R. Aloisio, P. Blasi, A. Galante and A.F. Grillo, Astrop. Phys. 20, 369 (2003).

32. N.R. Bruno, G. Amelino-Camelia and J. Kowalski-Glikman, Phys. Lett. B522 133 (2001). G. Amelino-Camelia, Int. J. Mod. Phys. D11 1643 (2002). G. Amelino-Camelia Nature 41834 (2002).

33. T. Tamaki, T. Harada, U. Miyamoto and T. Torii, Phys. Rev. D65 083003 (2002).

34. A. Camacho, Gen. Rel. Grav. 35319 (2003).

35. A. Kempf, G. Mangano and R.B. Mann, Phys. Rev. D52 1108 (1995). 